\title{
Analytical investigation of a figure-eight single-pulse all-fiber laser based on a nonlinear amplifying loop mirror
}

\author{
M. Salhi, F. Amrani, H. Leblond, and F. Sanchez \\ Laboratoire de Photonique d'Angers, EA 4464, UFR Sciences, Université d'Angers, 2 Boulevard Lavoisier, \\ FR-49045 Angers Cedex 01, France
}

(Received 21 June 2010; published 21 October 2010)

\begin{abstract}
We establish analytically a master equation of a figure-eight all-fiber passively mode-locked laser. The nonlinear amplifying loop mirror (NALM) is used as an effective saturable absorber in order to generate short pulses. The master equation is of the cubic complex Ginzburg-Landau type, in which the coefficients explicitly depend on the characteristics of the cavity, in particular on the orientation of the polarizer, the coupling coefficient, and the length of the NALM. Single-pulse and continuous-wave (cw) solutions in both normal and anomalous dispersion are discussed analytically. In the anomalous dispersion situation, the equation governing the evolution of the system admits stable analytic pulse solutions. The pulse duration and energy are studied. The analysis provides domains in the space of the cavity parameters where energetic soliton and ultrashort pulses are obtained.
\end{abstract}

DOI: 10.1103/PhysRevA.82.043834

PACS number(s): 42.55.Wd, 42.65.Tg

\section{INTRODUCTION}

During the last 20 years, passively mode-locked (ML) fiber lasers have became mature thanks to more efficient techniques with advanced technology. The ultrashort single and multiple pulse regimes are generated experimentally through the nonlinear polarization evolution (NPE) [1-5]. These studies considered a unidirectional ring cavity configuration with polarizer and polarization controllers. The optical Kerr effect of the fiber causes a large rotation of the polarization for high intensity, and consequently the polarizer and polarization controllers allow one to manage the nonlinear losses. Analytical [6-8] and numerical [9,10] studies have been developed in order to predict the laser operation. B. Ortaç et al. [11] demonstrated experimentally the generation of $265 \mathrm{~nJ}$ ultrashort pulses by employing a saturable absorber and ytterbium-doped short-length large-mode-area fiber.

The figure-eight cavity design involves two loops, one of which is unidirectional, while the other one, the loop mirror, allows propagation in both directions. Two different configurations are possible: the amplifying fiber may be inserted in either the first or second loop. If the amplifier is in the unidirectional ring, the loop mirror is passive and is called a nonlinear optical loop mirror (NOLM). If the amplifier is inserted in the other loop, the latter is called a nonlinear amplifying loop mirror (NALM). Many studies have proved the ability of figure-eight lasers to produce subpicosecond pulses. The first demonstration of the passively mode-locked laser with NALM was given by Duling [12]. In this experiment, the laser cavity was all fiber, and 2-ps pulse widths were measured. Pulses as short as $620 \mathrm{fs}$ have also been reported in a fiber laser operating at $1.3 \mu \mathrm{m}$ wavelength in a praseodymium fluoride fiber laser [13]. A self-starting mode locking has been observed in a laser configuration based on a symmetrical NOLM with a twisted, birefringent fiber and a quarter-wave retarder in the loop [14,15]. A completely fiber integrated figure-eight laser generating an $850 \mathrm{fs}$ pulse at $1065 \mathrm{~nm}$ is reported in [16]. Passive mode locking was also demonstrated experimentally in a polarization maintaining a figure-eight erbium-doped fiber laser [17]. In addition, the figure-eight geometry is able to operate in a multiple-pulse regime $[18,19]$.
The large number of dynamics behaviors of the figure-eight laser has led to the development of theoretical models. Haus and coworkers [20] presented a simple phenomenological analysis of the figure-eight laser based on the assumptions that the changes per pass are small and the medium is isotropic. The model does not allow one to determine the stability criteria of pulses with respect to the coupling coefficient of the coupler between the nonlinear optical loop (NOL) and NALM. The pulse characteristics were not discussed either. Numerical simulations reported by Theimer and Haus [21] demonstrate that the pulse width depends on several factors including the gain and length of the amplifier and the length of the NOLM. Calculations by Pottiez [22] show that high-energy pulses can be obtained with a figure-eight erbium-doped fiber laser with large normal net dispersion and intensity-dependent transmission created by the NOLM. A model equation of the cubic complex Ginzburg-Landau (CGL) type has been derived for a configuration using a NOLM, in which all coefficients were expressed in terms of the parameters of the cavity [23]. It brought forward the effect of the coupling coefficient on the laser behavior.

In the present work, we develop an analytical model for an all-fiber passively mode-locked laser built with a NALM in the figure-eight configuration. The system of equations which governs propagation in the anisotropic fiber is reduced, in the presence of polarizer, to a scalar cubic CGL equation. A remarkable fact is the explicit dependence of the coefficients of the equation on the characteristics of the cavity. The domains of either mode-locking or continuous-wave (cw) behavior in the positive group velocity dispersion (GVD) are given versus both the coupling coefficient of the fiber coupler connecting the NOL with the NALM and the orientation of the polarizer. The characteristics of the soliton are investigated in anomalous dispersion. This work is the complement of the investigation presented in our previous work concerning the passively modelocked laser generated by the NOLM in the figure-eight cavity [23].

The laser configuration under consideration is presented in Fig. 1. It consists of a NALM connected with a NOL by a variable fiber coupler with a coupling coefficient $k$. The NOL 


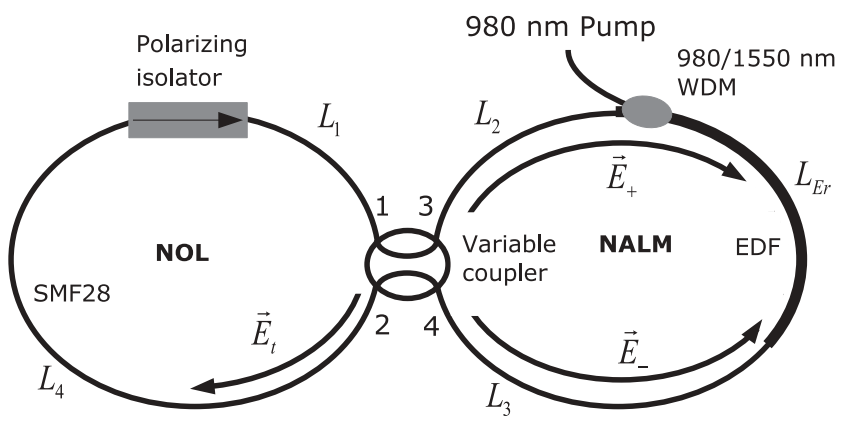

FIG. 1. Configuration of figure-eight fiber laser.

is constructed with a polarization-sensitive isolator, a standard communication fiber (SMF28) of length $L_{1}=1 \mathrm{~m}$ placed between the isolator and port 1 of the coupler, and a piece of SMF28 $\left(L_{4}=10 \mathrm{~m}\right)$ between port 2 of the coupler and the isolator. The polarizing isolator is used to obtain a travelingwave laser. Single-mode fibers have the following parameters: GVD $\beta_{2}^{s}=-0.022 \mathrm{ps}^{2} \mathrm{~m}^{-1}$ at $1550 \mathrm{~nm}$, nonlinear coefficient $\gamma=0.002 \mathrm{~W}^{-1} \mathrm{~m}^{-1}$, and birefringent parameter $n_{s}=1 \mathrm{~m}^{-1}$. The NALM involves a $L_{\mathrm{Er}}=10 \mathrm{~m}$ long erbium-doped fiber (EDF) with GVD $\beta_{2}^{\mathrm{Er}}=0.075 \mathrm{ps}^{2} \mathrm{~m}^{-1}$ at $1550 \mathrm{~nm}$, a nonlinear coefficient $\gamma=0.002 \mathrm{~W}^{-1} \mathrm{~m}^{-1}$, and a birefringent parameter $n_{\mathrm{Er}}=0.1 \mathrm{~m}^{-1}$. The erbium-doped fiber is inserted between two variable lengths $L_{2}$ and $L_{3}$ of SMF28. Wavelength division multiplexing (WDM) is used to launch the pump power into the erbium-doped fiber. In such a configuration, the mechanism responsible for the mode-locking regime is the intensity-dependent transmission created by NALM. The incoming beam is split into two beams which follow a trajectory in opposite directions. The pulse which enters the gain medium first undergoes a larger phase shift through self-phase modulation than its replica propagating in the opposite direction does. The two pulses arrive simultaneously at the fiber coupler where they recombine. Thus, the NALM behaves as a fast saturable absorber.

\section{NET POSITIVE DISPERSION}

We study in this section the figure-eight laser operating in normal dispersion. We use here the general method developed in [24]. We first solve the coupled nonlinear Schrödinger (NLS) equations describing an anisotropic standard fiber by using the perturbation method. The perturbation approach is also used to solve the equations governing the wave propagation in the erbium-doped fiber with distributed gain. In our analysis we take into account the polarizer effect and the fiber coupler action. The electric field at the exit of the polarizer at the $(n+1)$ th round trip is computed as a function of its amplitude at the $n$th round trip. To discuss the localized and stationary solutions, it is more convenient to transform the discrete sequence into a continuous equation of the CGL type.

\section{A. Equations of intracavity propagation of light}

\section{Propagation in the NALM}

In anisotropic optical fibers, the evolution of the two slowly varying envelopes $u$ and $v$ of the two polarization components of the electric field is described by means of a pair of coupled nonlinear Schrödinger equations. Each equation takes into account the birefringence, the GVD, and the Kerr nonlinearity in the case of a single-mode fiber. In the erbium-doped fiber, we consider in addition the gain and its bandwidth. Hence in the framework of the eigenaxis of the birefringent fiber moving at the group velocity, the pulse envelope propagation is modeled by the equations [24,25]

$$
\begin{aligned}
& i \frac{\partial u}{\partial z}-n_{j} u-\frac{\beta_{2}^{j}}{2} \frac{\partial^{2} u}{\partial t^{2}}+\gamma\left(u|u|^{2}+A u|v|^{2}+B v^{2} u^{*}\right) \\
& \quad=i g u+i \rho \frac{\partial^{2} u}{\partial t^{2}}, \\
& i \frac{\partial v}{\partial z}+n_{j} v-\frac{\beta_{2}^{j}}{2} \frac{\partial^{2} v}{\partial t^{2}}+\gamma\left(v|v|^{2}+A v|u|^{2}+B u^{2} v^{*}\right) \\
& \quad=i g u+i \rho \frac{\partial^{2} v}{\partial t^{2}},
\end{aligned}
$$

where $A=2 / 3$ and $B=1 / 3$ [25] are the dielectric coefficients, and $j=s$ in the standard fiber, or $j=\mathrm{Er}$ in the active fiber. The gain $g$ and, consequently, the gain filtering $\rho=g / \omega_{g}^{2}$ $\left(\omega_{g}=15.7 \mathrm{ps}^{-1}\right.$ is the spectral gain bandwidth) vanish in SMF28.

The propagation in the NALM is characterized by two counterpropagating fields denoted by $\mathbf{E}_{ \pm}=\left(u_{ \pm}, v_{ \pm}\right)$. The clockwise pulse $\mathbf{E}_{+}$circulating between port 3 and port 4 (see Fig. 1) propagates over the piece $L_{2}$ of SMF28, along the length $L_{\mathrm{Er}}$ of the EDF and along the length $L_{3}$ of SMF28. We denote it by $\mathbf{E}_{+}(0)=\left(u_{+, 0}, v_{+, 0}\right)$ at the entrance of the loop, just after port 3 , and at the exit of each piece of the fiber it leads to the electric-field components $\mathbf{E}_{+}\left(L_{2}\right)=\left(u_{+, 2}, v_{+, 2}\right), \mathbf{E}_{+}\left(L_{2}+\right.$ $\left.L_{\mathrm{Er}}\right)=\left(u_{+, \mathrm{Er}}, v_{+, \mathrm{Er}}\right)$, and $\mathbf{E}_{+}\left(L_{2}+L_{\mathrm{Er}}+L_{3}\right)=\left(u_{+, 3}, v_{+, 3}\right)$, respectively. The anticlockwise pulse $\mathbf{E}_{-}=\left(u_{-}, v_{-}\right)$propagates successively along the length $L_{3}, L_{\mathrm{Er}}$, and $L_{2}$, starting from $\mathbf{E}_{-}(0)=\left(u_{-, 0}, v_{-, 0}\right)$ just after port 4 , and yielding the electric-field components $\mathbf{E}_{-}\left(L_{3}\right)=\left(u_{-, 3}, v_{-, 3}\right), \mathbf{E}_{-}\left(L_{3}+\right.$ $\left.L_{\mathrm{Er}}\right)=\left(u_{-, \mathrm{Er}}, v_{-, \mathrm{Er}}\right)$, and $\mathbf{E}_{-}\left(L_{3}+L_{\mathrm{Er}}+L_{2}\right)=\left(u_{-, 2}, v_{-, 2}\right)$, respectively.

The components $\left(u_{ \pm, \mathrm{Er}}, v_{ \pm, \mathrm{Er}}\right)$ at the ends of the erbiumdoped fiber are computed analytically by solving Eqs. (1) and (2) by means of a first-order perturbative approach [6,7,24], assuming that the effect of GVD $\beta_{2}^{j}$, of the nonlinear coefficient $\gamma$, and of the gain filtering $\rho=g / \omega_{g}^{2}$ are small over one round trip in the cavity. The latter quantities are multiplied by a small parameter $\varepsilon$, and the solutions are sought in the form of series expansion in powers of $\varepsilon$. $\left(u_{ \pm, 2}, v_{ \pm, 2}\right)$ and $\left(u_{ \pm, 3}, v_{ \pm, 3}\right)$ are computed the same way, except that the right-hand-side terms in Eqs. (1) and (2) are replaced with zero. The explicit expressions are given in the Appendix.

The cross-phase modulation between the two counterpropagating fields is neglected in our calculations. Indeed, for short pulses, the two waves cross in a very short distance compared to the fiber loop length, which renders their interaction negligible. Hence the interaction terms $u_{+}\left|u_{-}\right|^{2}, u_{+}\left|v_{-}\right|^{2}$, $u_{-}^{2} u_{+}^{*}$, and $v_{-}^{2} u_{+}^{*}$ are not considered in the evolution equation for $u_{+}$, and so on.

\section{Fiber optic splitter and propagation in $\mathrm{NOL}$}

The electric field at the output of the polarizing isolator is $\mathbf{E}(0)=\left(u_{0}, v_{0}\right)$. It then evolves nonlinearly along the distance 
$L_{1}$ of SMF28 according to Eqs. (1) and (2) with zero gain and gain filtering. The resulting field $\mathbf{E}\left(L_{1}\right)=\left(u_{1}, v_{1}\right)$, obtained under the same approximations as above, is split by the fiber coupler into the two counterpropagating fields $\mathbf{E}_{ \pm}$whose evolution has been computed in the previous subsection. The light transmitted to ports 3 and 4 is written as a function of the light injected into port 1 as $\mathbf{E}_{+}(0)=\left(u_{+, 0}, v_{+, 0}\right)=\sqrt{k}\left(u_{1}, v_{1}\right)$ and $\mathbf{E}_{-}(0)=\left(u_{-, 0}, v_{-, 0}\right)=i \sqrt{1-k}\left(u_{1}, v_{1}\right)$, where $k$ is the coupling coefficient.

The field transmitted by the NALM results from the recombination at port 2 of the coupler of the two counterpropagating waves after one round trip in the loop containing the active fiber. It is given by

$$
\mathbf{E}_{t}(0)=\left(\begin{array}{c}
u_{t, 0} \\
v_{t, 0}
\end{array}\right)=\sqrt{k}\left(\begin{array}{c}
u_{+, 3} \\
v_{+, 3}
\end{array}\right)+i \sqrt{1-k}\left(\begin{array}{c}
u_{-, 2} \\
v_{-, 2}
\end{array}\right) .
$$

After propagation over the length $L_{4}$, the field $\mathbf{E}_{t}(0)$ becomes $\mathbf{E}_{t}\left(L_{4}\right)=\left(u_{t, 4}, v_{t, 4}\right)$ [see Eqs. (A15)-(A16) in the Appendix].

\section{The polarizer}

A polarizing isolator converts an elliptical or circular vibration into a beam with a linear polarization and stops the counterpropagating wave in the NOL. The presence of the polarizer in the cavity allows us to reduce the vectorial discrete evolution equation into a scalar discrete equation for the electric-field amplitude.

We describe the polarizer by the Jones formalism. We assume that the eigenaxis of the fiber ends, at each side of the polarizing isolator, are aligned and denote them as the $x$ and $y$ axes. The transmission axis of the polarizer makes an angle $\theta$ with the $x$ axis. The Jones matrix of the polarizer in the $(x, y)$ frame can be expressed as

$$
M=\beta\left(\begin{array}{ll}
\cos ^{2} \theta & \cos \theta \sin \theta \\
\cos \theta \sin \theta & \sin ^{2} \theta
\end{array}\right),
$$

where $\beta=95 \%$ is the amplitude transmission coefficient of the polarizer.

When the light passes through the polarizer, it oscillates with a linear polarization parallel to the passing axis of the polarizer. The resulting electric-field components at the beginning if the $n$th round trip at the input of the SMF28 fiber spliced with port 1 of the fiber coupler are thus

$$
\left(\begin{array}{l}
u_{n, 0} \\
v_{n, 0}
\end{array}\right)=\left(\begin{array}{c}
\cos \theta \\
\sin \theta
\end{array}\right) f_{n}
$$

where $f_{n}$ is the electric-field amplitude at the beginning of the $n$ round trip, just after the polarizer.

The electric field at the beginning of the $(n+1)$ th round trip, just after the polarizer, is found by taking the product of the Jones matrix of the polarizer and the Jones vector of the electric field at the end of the $n$ round trip $\mathbf{E}_{t, n}\left(L_{4}\right)=\left(u_{t, n, 4}, v_{t, n, 4}\right)$ at the exit of the SMF28 fiber with length $L_{4}$, as

$$
\left(\begin{array}{c}
\cos \theta \\
\sin \theta
\end{array}\right) f_{n+1}=M\left(\begin{array}{c}
u_{t, n, 4} \\
v_{t, n, 4}
\end{array}\right) \text {. }
$$

\section{B. Equation governing wave propagation for the figure-eight laser}

The master equation governing the propagation in the figure-eight laser is found by first reporting the expressions (A15) and (A16) of $\left(u_{t, n, 4}, v_{t, n, 4}\right)$ into Eq. (6). Then relation (3) is used, and $f_{n+1}$ is related to the clockwise and anticlockwise fields after propagation in NALM. We use the solutions of (A1) to (A6) and of (A7) to (A12) in order to express the components $\left(u_{+, n, 3}, v_{+, n, 3}\right)$ and $\left(u_{-, n, 2}, v_{-, n, 2}\right)$ as functions of $\left(u_{+, n, 0}, v_{+, n, 0}\right)$ and $\left(u_{-, n, 0}, v_{-, n, 0}\right)$, respectively. Then we replace the last two vectors by $\sqrt{k}\left(u_{n, 1}, v_{n, 1}\right)$ and $i \sqrt{1-k}\left(u_{n, 1}, v_{n, 1}\right)$, respectively. Finally, Eqs. (A13) and (A14) allows us to relate $f_{n+1}$ to $\left(u_{n, 0}, v_{n, 0}\right)$, and using Eq. (5), to $f_{n}$ itself. After lengthy and cumbersome algebra, we obtain

$$
\begin{aligned}
f_{n+1}= & \beta e^{g L_{\mathrm{Er}}} Q f_{n}+\varepsilon \beta e^{g L_{\mathrm{Er}}} \\
& \times\left[\left(\chi-\frac{i}{2} \eta\right) Q \frac{\partial^{2} f_{n}}{\partial t^{2}}+i P f_{n}\left|f_{n}\right|^{2}\right]+O\left(\varepsilon^{2}\right),
\end{aligned}
$$

where $\chi=\rho L_{\mathrm{Er}}, \quad \eta=\beta_{2}^{s}\left(L_{1}+L_{2}+L_{3}+L_{4}\right)+\beta_{2}^{\mathrm{Er}} L_{\mathrm{Er}}=$ $\beta_{2}^{s} L_{s}+\beta_{2}^{\mathrm{Er}} L_{\mathrm{Er}}$ is the net dispersion, and

$$
Q=(2 k-1)\left(e^{-i n_{s} L_{s}-i n_{\mathrm{Er}} L_{\mathrm{Er}}} \cos ^{2} \theta+e^{i n_{s} L_{s}+i n_{\mathrm{Er}} L_{\mathrm{Er}}} \sin ^{2} \theta\right) .
$$

$P$ is a complex parameter and represents the nonlinearity; its expression is not given here because of its excessive length. $P$ and $Q$ depend, especially, on the coupling coefficient $k$ of the fiber coupler, on the orientation $\theta$ of the polarizing isolator, and on the lengths $L_{2}$ and $L_{3}$ of SMF28.

Equation (7) allows us to calculate the gain threshold $g_{0}$. The gain threshold is obtained when the optical gain of the laser medium is exactly balanced by the sum of all losses experienced by the light in the cavity. At threshold, the intensity is low, and gain and losses are purely linear. This situation, which corresponds to the conservation of intensity, expresses mathematically by requiring that the equation $\left|f_{n+1}\right|^{2}=\left|f_{n}\right|^{2}$ is satisfied at leading order in $\varepsilon$. Therefore the gain is written as $g=g_{0}+\varepsilon g_{1}$. The excess of linear gain $g_{1}$ will compensate the nonlinear losses and is self-adjusted [24].

The expression of $g_{0}$ is

$$
\begin{aligned}
g_{0}= & \frac{-1}{2 L_{\mathrm{Er}}} \ln \left\{\beta ^ { 2 } ( 2 k - 1 ) ^ { 2 } \left[\cos ^{4} \theta+2 \cos \left(2 L_{s} n_{s}+2 L_{\mathrm{Er}} n_{\mathrm{Er}}\right)\right.\right. \\
& \left.\left.\times \cos ^{2} \theta \sin ^{2} \theta+\sin ^{4} \theta\right]\right\} .
\end{aligned}
$$

$g_{0}$ denotes the amount of the gain that compensates the linear losses of the laser cavity.

Since $\left|Q \beta e^{g_{0} L_{\mathrm{Er}}}\right|=1$, it can be replaced by $e^{i \alpha}$ with some $\alpha$ real. Then, expanding $e^{\varepsilon g_{1} L_{\mathrm{Er}}}$ in a power series of $\varepsilon$ and rearranging Eq. (7) yields

$$
\begin{aligned}
f_{n+1}= & e^{i \alpha}\left(1+\varepsilon g_{1} L_{\mathrm{Er}}\right) f_{n}+\varepsilon\left(\chi-\frac{i}{2} \eta\right) e^{i \alpha} \frac{\partial^{2} f_{n}}{\partial t^{2}} \\
& +i \varepsilon \frac{e^{i \alpha}}{Q} P f_{n}\left|f_{n}\right|^{2}+O\left(\varepsilon^{2}\right) .
\end{aligned}
$$


The discrete sequence $f_{n}$ describing the field amplitude does not allow us to use the known results on the CGL equation to treat the continuous and single-pulse operation of the laser. Therefore, the discrete sequence is interpolated, in the case of a large number of round trips in the cavity, by a continuous function, and we obtain

$$
\begin{aligned}
i \frac{\partial f}{\partial z}= & \frac{-\alpha-i \ln (2 k-1)}{L_{0}} f+i \varepsilon \frac{g_{1} L_{\mathrm{Er}}}{L_{0}} f \\
& +\varepsilon\left(\frac{\eta / 2+i \chi}{L_{0}}\right) \frac{\partial^{2} f}{\partial t^{2}}+\varepsilon \mathcal{D} f|f|^{2},
\end{aligned}
$$

where $z=n\left(L_{s}+L_{\mathrm{Er}}\right)=n L_{0}$ is the longitudinal variable and

$$
\mathcal{D}=\mathcal{D}_{r}+i \mathcal{D}_{i}=\frac{-P}{Q\left[L_{0}+4 k(k-1) L_{4}\right]} .
$$

The solution at zero-order perturbation of Eq. (11) for $k \neq 0.5$ is

$$
f=F \exp \left(\frac{i z \alpha-z \ln (2 k-1)}{L_{0}}\right)+O(\varepsilon) .
$$

We make the change of variable $\xi=\varepsilon z$ according to the multiscale analysis [26,27]. The space derivative operator becomes $\partial_{z}=\partial_{\hat{z}}+\varepsilon \partial_{\xi}$. The fast space variation is $\hat{z}$ and the slow space variation is $\xi$. The values of $\xi / L_{0}$ about 1 correspond to the number of round trips $n$ about $1 / \varepsilon$, or to a propagation distance $z=L_{0} / \varepsilon$, in which $L_{0}=L_{s}+L_{\mathrm{Er}}$ is the total cavity length, with $L_{s}=L_{1}+L_{2}+L_{3}+L_{4}$ the total length of SMF28. The variation of the amplitude $F$ with respect to the slow variable $\xi$ is obtained by requiring that the first-order correction $O(\varepsilon)$ evolves slower than linearly with respect to the fast variable $\hat{z}$. It finally yields the cubic CGL equation

$$
i \frac{\partial F}{\partial \xi}=i \frac{g_{1} L_{\mathrm{Er}}}{L_{0}} F+\frac{\eta / 2+i \chi}{L_{0}} \frac{\partial^{2} F}{\partial t^{2}}+\left(\mathcal{D}_{r}+i \mathcal{D}_{i}\right) F|F|^{2} .
$$

Here $\mathcal{D}_{r}$ is the effective self-phase modulation, it is negative. $\mathcal{D}_{i}$ is the effective nonlinear gain. When it is negative, it corresponds to an effective absorption. Note that $\mathcal{D}_{r}$ and $\mathcal{D}_{i}$ are explicitly computed as functions of the physical parameters of the laser, especially the orientation of the polarizer $\theta$, the coupling coefficient $k$, and the different lengths of fiber that make up the NALM.

\section{Solutions of the cubic complex Ginzburg-Landau equation}

We will restrict ourselves to the study of two particular solutions of Eq. (14): the stationary one, and the analytical soliton solution. The first one is the stationary solution corresponding to a $\mathrm{cw}$ operating regime of the laser. We analyze the existence and stability criterion. We then investigate the localized solution. The latter informs us about the single-pulse regime.

\section{Stationary solution}

The solution with constant modulus expresses as

$$
F=\Gamma e^{i(\kappa \xi-\Omega t)}
$$

where

$$
\begin{gathered}
\Omega^{2}=\frac{\mathcal{D}_{i}|\Gamma|^{2} L_{0}+g_{1} L_{\mathrm{Er}}}{\chi}, \\
\kappa=\frac{\eta}{2 \chi}\left(\mathcal{D}_{i}|\Gamma|^{2}+\frac{g_{1} L_{\mathrm{Er}}}{L_{0}}\right)-\mathcal{D}_{r}|\Gamma|^{2} .
\end{gathered}
$$

The solution (15) is independent of time when the temporal phase shift $\Omega$ is zero. Therefore, the expressions of $\Gamma$ and $\kappa$ are

$$
\Gamma=\sqrt{\frac{-g_{1} L_{\mathrm{Er}}}{\mathcal{D}_{i} L_{0}}}, \quad \kappa=\frac{g_{1} \mathcal{D}_{r} L_{\mathrm{Er}}}{\mathcal{D}_{i} L_{0}} .
$$

The solution (15) makes sense only if the constant amplitude $\Gamma$ is real, which means that the product $\mathcal{D}_{i} g_{1}$ must be negative. Moreover, the studies carried out in [24] show that modulational instability occurs when the excess of linear gain $g_{1}$ is negative and the nonlinear gain $\mathcal{D}_{i}$ is positive. As a result, the stationary solution is stable if

$$
g_{1}>0 \text { and } \mathcal{D}_{i}<0,
$$

i.e., the stability criterion for the $\mathrm{cw}$ behavior corresponds to a positive excess of linear gain and a negative effective nonlinear gain.

\section{Localized solution}

The dissipative localized solution to the CGL equation (14) is

$$
F=a(t)^{1+i d} e^{-i \omega \xi} .
$$

Here, $d$ is the chirp parameter, expressed as

$$
d=\frac{-3\left(\eta \mathcal{D}_{r}+2 \chi \mathcal{D}_{i}\right)+\sqrt{9\left(2 \chi \mathcal{D}_{i}+\eta \mathcal{D}_{r}\right)^{2}+8\left(\eta \mathcal{D}_{i}-2 \chi \mathcal{D}_{r}\right)^{2}}}{2\left(\eta \mathcal{D}_{i}-2 \chi \mathcal{D}_{r}\right)}
$$

while the frequency shift is

$$
\omega=\frac{-g_{1} L_{\mathrm{Er}}\left(4 \chi d+\eta d^{2}-\eta\right)}{2 L_{0}\left(\chi d^{2}-\chi-\eta d\right)} .
$$

$a(t)$ gives the hyperbolic secant shape of the pulse, as

$$
a(t)=M N \operatorname{sech}(M t)
$$

where

$$
\begin{gathered}
M=\sqrt{\frac{g_{1} L_{\mathrm{Er}}}{\chi d^{2}-\chi-\eta d}}, \\
N=\sqrt{\frac{3 d\left(4 \chi^{2}+\eta^{2}\right)}{2 L_{0}\left(\eta \mathcal{D}_{i}-2 \chi \mathcal{D}_{r}\right)}} .
\end{gathered}
$$


The hyperbolic secant pulse exists if the factor $M N$ is real. Consequently, the stability condition of the localized solution is deduced from Eq. (24). Indeed, $M$ is real if $g_{1}$ and the quantity $\left(\chi d^{2}-\chi-\eta d\right)$ have the same sign. In the defocusing case, where $\eta \mathcal{D}_{r}<0$, the hyperbolic secant pulse is never stable from the mathematical point of view. However, numerical resolution shows that if the excess of linear gain $g_{1}$ is negative and the effective nonlinear gain $\mathcal{D}_{i}$ is positive, then the pulses are self-starting and arise spontaneously from noise. Because the CGL equation does not take into account the gain saturation, the number of pulses may grow up indefinitely. Any refinement of the model taking into account the gain saturation and the fact that the energy in the cavity is bounded from above will stabilize the pulse train. Consequently, this situation corresponds to stable mode locking, and the stability criterion for mode locking is

$$
\left(\chi d^{2}-\chi-\eta d\right)<0 .
$$

Note that the bistability between single-pulse and continuous regime is not excluded when the effective nonlinear gain is negative.

More specifically, the inclusion of quintic nonlinear terms in the CGL equation (14) yields the so-called cubic-quintic CGL equation, which possesses stable pulse solutions [28]. Such additional quintic terms have been derived in the case of the fiber laser mode locked by nonlinear rotation of the polarization in [29], by considering the terms of order $\varepsilon^{2}$ in the perturbative expansion. Insofar as the perturbation approach is valid, the coefficients of the quintic terms remain small. As the effective quintic nonlinear index and the effective nonlinear gain-absorption coefficient (usually denoted by $v$ and $\mu$, respectively) tend to zero, the analytic fixed-amplitude solution to the cubic-quintic CGL equation tends to solution (20) to the cubic CGL equation [28]. The condition $g_{1}<0$ is still required for stability, and consequently condition (26) is valid as a stability condition for the analytic fixed-amplitude solution to the cubic-quintic CGL equation, in the limit of small values of the coefficients of the quintic terms.

The validity of the perturbative procedure has been checked in [29] for the fiber laser mode locked by nonlinear rotation of the polarization, by comparing the results of the resolution of the cubic-quintic CGL model with the full numerical resolution of the equations governing light propagation in the laser cavity. Quantitative agreement was found. In the present case of the figure-8 laser, the full numerical resolution of the propagation equations is a much more lengthy and complicated task, and we shall not perform it. Notice that to conclude on the nature of the laser behavior, only qualitative agreement is required.

\section{Results and discussion}

In the above section, we have given the conditions of existence and stability for the $\mathrm{cw}$ and mode-locked regimes. It is now possible to study the stability domains in the $(\theta, k)$ plane. The length of the NALM also plays an important role in the distribution of regimes in the $(\theta, k)$ plane. For this reason, we varied $L_{2}$ and $L_{3}$. The length of the active fiber remains constant.

To treat the effect of the NALM length on the regime distribution in the $(\theta, k)$ plane, we proceed as follows. We (a)

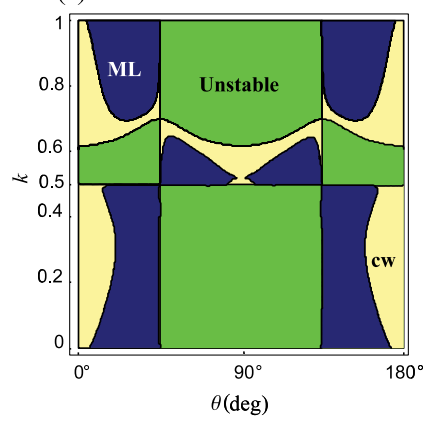

(b)

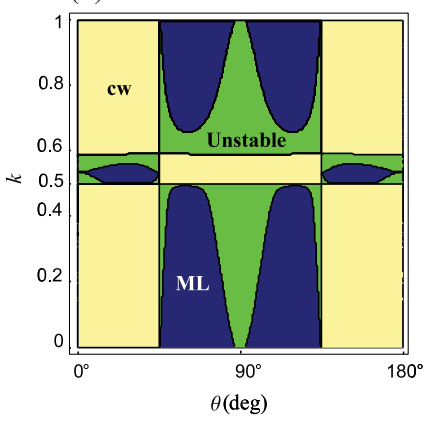

FIG. 2. (Color online) Stability diagram of the $\mathrm{cw}$ and $\mathrm{ML}$ solutions for (a) $L_{2}=3 \mathrm{~m}, L_{3}=2 \mathrm{~m}$ and (b) $L_{2}=7.5 \mathrm{~m}, L_{3}=5 \mathrm{~m}$ in the plane $(\theta, k)$. The yellow (light gray) region corresponds to stable $\mathrm{cw}$ and TXtable unstable ML operation, the blue (black) region corresponds to stable ML and unstable cw operation. The green (dark gray) corresponds to unstable cw and unstable ML operation.

first fix the length $L_{3}$ equal to zero and vary $L_{2}$ from 0 to $23 \mathrm{~m}$ by steps of $0.5 \mathrm{~m}$. We took subsequently $L_{3}=1,2,3,4$, and $5 \mathrm{~m}$, and for each value we varied $L_{2}$. Note that the total length is chosen in such a way that the net dispersion is still positive. In this situation, the criterion (26) is valid. The cartographies obtained reveal a great sensitivity to $\theta, k, L_{2}$, and $L_{3}$. Examples of diagrams are given in Fig. 2(a) for $L_{2}=3 \mathrm{~m}$ and $L_{3}=2 \mathrm{~m}$ and in Fig. 2(b) for $L_{2}=7.5 \mathrm{~m}$ and $L_{3}=5 \mathrm{~m}$. The operating regimes are not symmetric with respect to $k=0.5$ except for $L_{2}=L_{3}$. In some situations, however, a mode locking occurs only for $k>0.5$. Figure 3 shows a mapping corresponding to $L_{2}=7.5 \mathrm{~m}$ and $L_{3}=1 \mathrm{~m}$.

The analysis performed on the cartographies showed a disappearance of the pulsed regime for some particular values of lengths $L_{2}$ and $L_{3}$. This observation was expected because the length of the NALM affects the total nonlinear phase shift between clockwise and anticlockwise propagating pulses. Interference between these two pulses gives a low contrast between high and low intensities. The resulting pulse in port 2 is larger than the input pulse in port 1 and makes mode-locked operation of the laser unstable. The cartographies also demonstrate that if the light is linearly polarized and

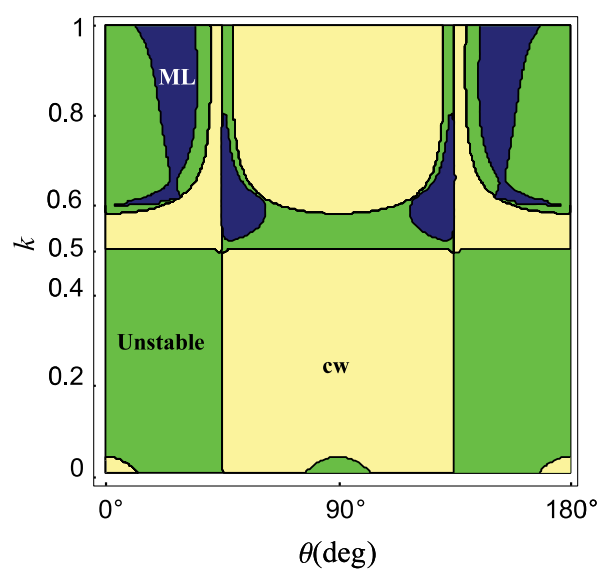

FIG. 3. (Color online) Stability diagram of the $\mathrm{cw}$ and ML solutions for $L_{2}=7.5 \mathrm{~m}$ and $L_{3}=1 \mathrm{~m}$ in the plane $(\theta, k)$. The colors have the same meaning as in Fig. 2. 
parallel to one of the eigenaxis of the entrance of fiber of length $L_{1}\left(\theta=0^{\circ}\right.$ or $\left.90^{\circ}\right)$ or circularly polarized $\left(\theta=45^{\circ}\right.$ or $135^{\circ}$ ), then the localized solutions are unstable. This observation is in agreement with experimental and numerical results reported by Stentz and Boyd [30]. The authors showed that the polarization plays an important role in figure-eight fiber lasers. However, contrary to our model, the work did not specify the polarizations leading to instability of the modelocked regime. In addition, according to our calculations, the latter regime becomes unstable near zero net dispersion $\eta=\beta_{2}^{s} L_{s}+\beta_{2}^{\mathrm{Er}} L_{\mathrm{Er}} \simeq 0^{+}$[31]. Indeed, in the presence of an excess of linear gain $g_{1}$ and an effective nonlinear gain $\mathcal{D}_{i}$, the pulse tends to grow dramatically.

The growth is equilibrated by nonlinear self-defocusing. The latter requires both Kerr effect and dispersion. In the absence of dispersion, there is no self-defocusing and the pulse cannot be stabilized. If higher-order dispersion terms are taken into account in the propagation equation, self-defocusing can be recovered, and consequently pulse stabilization can occur.

\section{NET NEGATIVE DISPERSION}

\section{A. Stability of stationary and localized solutions}

We discuss in this section a situation corresponding to a net negative dispersion $\eta=\beta_{2}^{s} L_{s}+\beta_{2}^{\mathrm{Er}} L_{\mathrm{Er}}<0$. In such case, lengths $L_{2}$ and $L_{3}$ are adjusted in such a way that the figure-eight laser operates in the so-called soliton regime. It is worth noticing that the previously established equations remain valid, except the stability criterion of the pulsed regime. The expression and the stability criterion of the constant solution are not sensitive to the sign of dispersion.

To determine the criterion of existence and stability of solution (20) in the case of net negative dispersion and positive nonlinear gain $\mathcal{D}_{i}$, we consider the criterion established by Akhmediev et al. [32]. They found that the soliton (20) is stable when the denominator in the expression for $M$ is positive. The excess of linear gain $g_{1}$ is positive and the zero solution $(F \sim 0)$ is unstable.

$$
\left(\chi d^{2}-\chi-\eta d\right)>0 .
$$

On the $\left(\mathcal{D}_{i}, \chi\right)$ plane, the solution is stable below the curve

$$
\mathcal{D}_{i}=\mathcal{D}_{i}^{S}=-\chi \mathcal{D}_{r} \frac{3 \sqrt{\eta^{2}+4 \chi^{2}}+\eta}{4 \eta^{2}+18 \chi^{2}},
$$

and unstable above this curve. Since the pulse solution of the cubic CGL equation is stable, additional quintic terms are not required to describe the laser behavior, in contrast to the case of normal dispersion. We point out that, in our model, the coefficients of the CGL equation explicitly depend on the characteristics of the laser, hence condition (27) expresses in terms of laser parameters. It should be emphasized that when the nonlinear gain $\mathcal{D}_{i}$ is negative, the stability condition is unknown at this time. The continuous laser emission corresponding to a stable stationary solution occurs in this case for a positive excess of linear gain $g_{1}$. In addition, the competition between continuous and mode-locked emission could be envisaged. Finally, it is important to note that for positive nonlinear gain and positive excess of linear gain, the localized solution can be distorted for long-distance propagation by the instability developed by the background state.

\section{B. Pulse energy}

In the present focusing case, the hyperbolic secant pulse solution (21)-(26) to CGL is stable from the mathematical point of view, and hence it represents correctly the physical laser pulse in the cavity. Therefore, it is possible to use the analytical expression of the pulse to investigate its energy as a function of $k, \theta$, and NALM length. In a homogeneously broadened gain medium, the saturated gain can be written as

$$
g_{0}=\frac{g^{\prime}}{1+\left(E / E_{S}\right)},
$$

where $g^{\prime}=1.26 \mathrm{~m}^{-1}$ is the unsaturated gain, $E_{S}=0.1 \mathrm{pJ}$ the saturation energy, and $E$ the pulse energy. This saturated gain is also the gain which compensates the linear losses, $g_{0}$, given in Eq. (9). It is now easy through Eq. (29) to deduce the expression of energy.

The inspection of the single-pulse soliton energy in the plane $(\theta, k)$ versus the variation of the length of NALM is performed in the soliton regime. For this condition, $L_{2}+L_{3}$ must be greater than $24 \mathrm{~m}$. We have thus taken $L_{3}=24 \mathrm{~m}$ and varied $L_{2}$ from 0 to $20 \mathrm{~m}$ with a step of $0.5 \mathrm{~m}$. The interesting point of our analytical simulations is that the most energetic solitons are always obtained with a highly asymmetrical coupler and close to alignment between the polarizer and the fast or slow axes at the entrance of fiber placed in port 1 of the variable coupler (corresponding, respectively, to $\theta=0^{\circ}$ or $90^{\circ}$ ). The regions with mode-locking energy greater than $2 \mathrm{pJ}$ are small and occur if the coupling coefficient $k$ of the coupler is below 0.2 or above 0.8 . Such zones are observed when the polarizer is aligned with the fast axis of the entrance of the fiber of length $L_{1}$. No energies greater than $2 \mathrm{pJ}$ were observed in the $(\theta, k)$ plane for an alignment between the polarizer and the slow axis of the entrance of fiber of length $L_{1}$, except for $L_{2}$ equal to $9.5 \mathrm{~m}$. The opposite situation is also observed. An example of cartography is represented in Fig. 4 for $L_{2}=19 \mathrm{~m}$ and $L_{3}=24 \mathrm{~m}$. The maximum energy is about $24 \mathrm{pJ}$; it is obtained for $\theta=90^{\circ}$, and $k$ close to 0 or $k$ close to 1 . If $L_{2}=9.5 \mathrm{~m}$, the highest energy corresponds to $\theta=0^{\circ}$ (or $180^{\circ}$ ) and $k$ close to 1 , or $\theta=90^{\circ}$ and $k$ close to 0 . From the experimental point of view, the insertion of a simple polarization controller after the polarizer allows the rotation of the polarization and the alignment between the latter and one of the principal axes of the input fiber of length $L_{1}$.

\section{Pulse duration}

Our analytical approach is also able to determine the pulse duration in the soliton regime. The pulse width is correlated to the pulse energy through the parameter $M$. Since the pulse width is $t_{0}=1 / M$, it depends on the parameter $M$, which is a function of the excess of linear gain $g_{1}$, the coupling coefficient $k$, the orientation of the polarizer, and the length of the nonlinear amplifying loop mirror. Since the excess of linear gain $g_{1}$ is self-adjusted and is not known in our model, for exploring the variations of $t_{0}$ in the plane $(\theta, k)$, we need some other expression of $M$, which do not involve $g_{1}$. Such an 


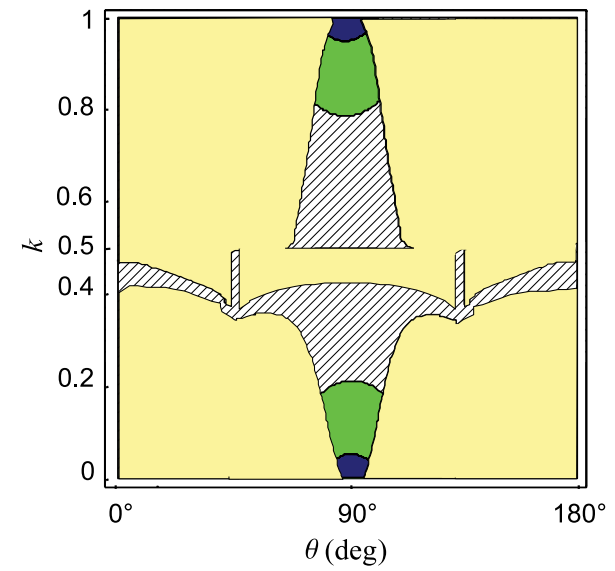

FIG. 4. (Color online) Variation of pulse energy in the $(\theta, k)$ plane for $L_{2}=19 \mathrm{~m}$ and $L_{3}=24 \mathrm{~m}$. In the blue (black) region, the energy is above $8 \mathrm{pJ}$; in the green (dark gray) region, the energy is between 2 and $8 \mathrm{pJ}$; and in the hatched region, the energy is below $2 \mathrm{pJ}$. In the yellow (light gray) region, either the pulses are unstable or their stability is not determined and continuous emission occurs.

expression can be drawn from the pulse energy $E=\int|F|^{2} d t$. After some calculation, we obtain

$$
E=2 M N^{2} .
$$

The pulse duration is thus

$$
t_{0}=\frac{2 N^{2}}{E}
$$

which shows explicitly that it is inversely proportional to the energy.

The latter can be computed from the expression of the saturated gain (29) as $E=\left(g^{\prime} / g_{0}-1\right) E_{S}$, where $N$ is given by (25) and the gain threshold $g_{0}$ by (9), both of which do not involve $g_{1}$. Through these quantities, the pulse width is also affected by the total dispersion in the cavity $\eta$ and by nonlinear effects corresponding to the effective self-phase modulation $\mathcal{D}_{r}$ and the effective nonlinear absorption $-\mathcal{D}_{i}$.

In order to explore the influence of the length of NALM on the pulse duration in the $(\theta, k)$ plane, we proceed in the same way as for studying the pulse energy. $L_{2}$ has been varied between 0 and $20 \mathrm{~m}$ by a step of $0.5 \mathrm{~m}$ and $L_{3}$ has been fixed to $24 \mathrm{~m}$. Figure 5 shows an example of cartography for $L_{2}=19 \mathrm{~m}$ and $L_{3}=24 \mathrm{~m}$. The most important observation is that the regions of ultrashort pulses are not obtained only for the highly asymmetrical coupler, but also for the intermediate coupling coefficient $(k \simeq 0.35-0.6)$. In addition, a large range of orientation angles $(\theta)$ can be chosen to produce ultrashort pulses $\left(\theta\right.$ varies from $25^{\circ}$ to $\left.155^{\circ}\right)$. Note that the zones where the pulse width is shorter than $500 \mathrm{fs}$ are small compared with the regions where the pulses are unstable or where their stability is not determined. From the experimental point of view, the generation of ultrashort single pulses from a figureeight laser based on the NALM requires the optimization of the parameters of the cavity, in particular the orientation of the polarizer, the coupling coefficient, and the length of the NALM.

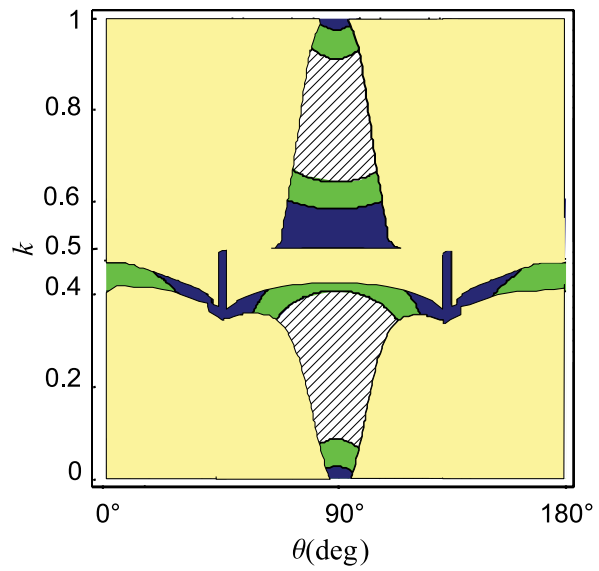

FIG. 5. (Color online) Evolution of the pulse duration in the $(\theta, k)$ plane for $L_{2}=19 \mathrm{~m}$ and $L_{3}=24 \mathrm{~m}$. In the hatched region, the duration is above $1 \mathrm{ps}$; in the green (dark gray) region, it is between 0.5 and $1 \mathrm{ps}$; and in the blue (black) region, below $0.5 \mathrm{ps}$. The yellow (light gray) regions have the same meaning as in Fig. 4.

\section{CONCLUSION}

We have established theoretically the master equation valid for both normal and anomalous dispersion regimes for a figure-eight single-pulse all-fiber laser based on NALM. The master equation of CGL type has been derived from two coupled nonlinear Schrödinger equations describing the evolution of the electric-field amplitude in the fiber. The coefficients of the equation are directly related to the characteristics of the laser cavity. In the normal dispersion case, we have presented the regions of stability of the ML and cw regimes in the $(\theta, k)$ plane. The effect of the length of the NALM was also pointed out. In the anomalous dispersion regime, thanks to its stability, the analytical solution describes well the real pulse, and consequently the pulse duration and energy have been investigated. The model predicts that the most energetic solitons occur for the high asymmetrical coupler and alignment between the polarizer and the fast or slow axes at the entrance of the fiber placed after the polarizer. Concerning the ultrashort single pulses, they may also exist for the intermediate coupling coefficient.

\section{APPENDIX}

This appendix lists the analytic solutions of the propagation equations for each piece of fiber.

\section{Clockwise pulse propagation in NALM}

Hereafter are the expressions of the copropagating wave electric field $\mathbf{E}_{+}=\left(u_{+}, v_{+}\right)$after propagation in each piece of fiber composing the NALM:

$$
\begin{aligned}
u_{+, 2}= & u_{+, 0} e^{-i n_{s} L_{2}}+\varepsilon\left[-\frac{i \beta_{2}^{s}}{2} L_{2} \frac{\partial^{2} u_{+, 0}}{\partial t^{2}}\right. \\
& +i \gamma L_{2}\left(u_{+, 0}\left|u_{+, 0}\right|^{2}+A u_{+, 0}\left|v_{+, 0}\right|^{2}\right) \\
& \left.+\frac{\gamma B}{4 n_{s}}\left(e^{4 i n_{s} L_{2}}-1\right) v_{+, 0}^{2} u_{+, 0}^{*}\right] e^{-i n_{s} L_{2}}+O\left(\varepsilon^{2}\right),
\end{aligned}
$$




$$
\begin{aligned}
& v_{+, 2}=v_{+, 0} e^{-i n_{s} L_{2}}+\varepsilon\left[-\frac{i \beta_{2}^{s}}{2} L_{2} \frac{\partial^{2} v_{+, 0}}{\partial t^{2}}\right. \\
& +i \gamma L_{2}\left(v_{+, 0}\left|v_{+, 0}\right|^{2}+A v_{+, 0}\left|u_{+, 0}\right|^{2}\right) \\
& \left.+\frac{\gamma B}{4 n_{s}}\left(e^{4 i n_{s} L_{2}}-1\right) u_{+, 0}^{2} v_{+, 0}^{*}\right] e^{-i n_{s} L_{2}}+O\left(\varepsilon^{2}\right), \\
& u_{+, \mathrm{Er}}=u_{+, 2} e^{\left(g-i n_{\mathrm{Er}}\right) L_{\mathrm{Er}}}+\varepsilon\left[L_{\mathrm{Er}}\left(\rho-\frac{i \beta_{2}^{\mathrm{Er}}}{2}\right) \frac{\partial^{2} u_{+, 2}}{\partial t^{2}}\right. \\
& +i \gamma\left(u_{+, 2}\left|u_{+, 2}\right|^{2}+A u_{+, 2}\left|v_{+, 2}\right|^{2}\right) \frac{e^{2 g L_{\mathrm{Er}}}-1}{2 g} \\
& \left.+i \gamma B v_{+, 2}^{2} u_{+, 2}^{*} \frac{e^{\left(2 g+4 i n_{\mathrm{Er}}\right) L_{\mathrm{Er}}}-1}{\left(2 g+4 i n_{\mathrm{Er}}\right)}\right] e^{\left(g-i n_{\mathrm{Er}}\right) L_{\mathrm{Er}}}+O\left(\varepsilon^{2}\right) \\
& v_{+, \mathrm{Er}}=v_{+, 2} e^{\left(g-i n_{\mathrm{Er}}\right) L_{\mathrm{Er}}}+\varepsilon\left[L_{\mathrm{Er}}\left(\rho-\frac{i \beta_{2}^{\mathrm{Er}}}{2}\right) \frac{\partial^{2} v_{+, 2}}{\partial t^{2}}\right. \\
& +i \gamma\left(v_{+, 2}\left|v_{+, 2}\right|^{2}+A v_{+, 2}\left|u_{+, 2}\right|^{2}\right) \frac{e^{2 g L_{\mathrm{Er}}}-1}{2 g} \\
& \left.+i \gamma B u_{+, 2}^{2} v_{+, 2}^{*} \frac{e^{\left(2 g+4 i n_{\mathrm{Er}}\right) L_{\mathrm{Er}}}-1}{\left(2 g+4 i n_{\mathrm{Er}}\right)}\right] e^{\left(g-i n_{\mathrm{Er}}\right) L_{\mathrm{Er}}}+O\left(\varepsilon^{2}\right) \text {, } \\
& u_{+, 3}=u_{+, \mathrm{Er}} e^{-i n_{s} L_{3}}+\varepsilon\left[-\frac{i \beta_{2}^{s}}{2} L_{3} \frac{\partial^{2} u_{+, \mathrm{Er}}}{\partial t^{2}}\right. \\
& +i \gamma L_{3}\left(u_{+, \mathrm{Er}}\left|u_{+, \mathrm{Er}}\right|^{2}+A u_{+, \mathrm{Er}}\left|v_{+, \mathrm{Er}}\right|^{2}\right) \\
& \left.+\frac{\gamma B}{4 n_{s}}\left(e^{4 i n_{s} L_{3}}-1\right) v_{+, \mathrm{Er}}^{2} u_{+, \mathrm{Er}}^{*}\right] e^{-i n_{s} L_{3}}+O\left(\varepsilon^{2}\right), \\
& v_{+, 3}=v_{+, \mathrm{Er}} e^{-i n_{s} L_{3}}+\varepsilon\left[-\frac{i \beta_{2}^{s}}{2} L_{3} \frac{\partial^{2} v_{+, \mathrm{Er}}}{\partial t^{2}}\right. \\
& +i \gamma L_{3}\left(v_{+, \mathrm{Er}}\left|v_{+, \mathrm{Er}}\right|^{2}+A v_{+, \mathrm{Er}}\left|u_{+, \mathrm{Er}}\right|^{2}\right) \\
& \left.+\frac{\gamma B}{4 n_{s}}\left(e^{4 i n_{s} L_{3}}-1\right) u_{+, \mathrm{Er}}^{2} v_{+, \mathrm{Er}}^{*}\right] e^{-i n_{s} L_{3}}+O\left(\varepsilon^{2}\right),
\end{aligned}
$$

\section{Anticlockwise pulse propagation in NALM}

The solutions of the counterpropagating wave electric field $\mathbf{E}_{-}=\left(u_{-}, v_{-}\right)$after propagation in each piece of fiber in the NALM are given by

$$
\begin{aligned}
u_{-, 3}= & u_{-, 0} e^{-i n_{s} L_{3}}+\varepsilon\left[-\frac{i \beta_{2}^{s}}{2} L_{3} \frac{\partial^{2} u_{-, 0}}{\partial t^{2}}\right. \\
& +i \gamma L_{3}\left(u_{-, 0}\left|u_{-, 0}\right|^{2}+A u_{-, 0}\left|v_{-, 0}\right|^{2}\right) \\
& \left.+\frac{\gamma B}{4 n_{s}}\left(e^{4 i n_{s} L_{3}}-1\right) v_{-, 0}^{2} u_{-, 0}^{*}\right] e^{-i n_{s} L_{3}}+O\left(\varepsilon^{2}\right), \\
v_{-, 3}= & v_{-, 0} e^{-i n_{s} L_{3}}+\varepsilon\left[-\frac{i \beta_{2}^{s}}{2} L_{3} \frac{\partial^{2} v_{-, 0}}{\partial t^{2}}\right. \\
& +i \gamma L_{3}\left(v_{-, 0}\left|v_{-, 0}\right|^{2}+A v_{-, 0}\left|u_{-, 0}\right|^{2}\right) \\
& \left.+\frac{\gamma B}{4 n_{s}}\left(e^{4 i n_{s} L_{3}}-1\right) u_{-, 0}^{2} v_{-, 0}^{*}\right] e^{-i n_{s} L_{3}}+O\left(\varepsilon^{2}\right),
\end{aligned}
$$

$$
\begin{aligned}
u_{-, \mathrm{Er}}= & u_{-, 3} e^{\left(g-i n_{\mathrm{Er}}\right) L_{\mathrm{Er}}}+\varepsilon\left[L_{\mathrm{Er}}\left(\rho-\frac{i \beta_{2}^{\mathrm{Er}}}{2}\right) \frac{\partial^{2} u_{-, 3}}{\partial t^{2}}\right. \\
& +i \gamma\left(u_{-, 3}\left|u_{-, 3}\right|^{2}+A u_{-, 3}\left|v_{-, 3}\right|^{2}\right) \frac{e^{2 g L_{\mathrm{Er}}}-1}{2 g} \\
& \left.+i \gamma B v_{-, 3}^{2} u_{-, 3}^{*} \frac{e^{\left(2 g+4 i n_{\mathrm{Er}}\right) L_{\mathrm{Er}}}-1}{\left(2 g+4 i n_{\mathrm{Er}}\right)}\right] e^{\left(g-i n_{\mathrm{Er}}\right) L_{\mathrm{Er}}}+O\left(\varepsilon^{2}\right),
\end{aligned}
$$

$$
\begin{aligned}
v_{-, \mathrm{Er}}= & v_{-, 3} e^{\left(g-i n_{\mathrm{Er}}\right) L_{\mathrm{Er}}}+\varepsilon\left[L_{\mathrm{Er}}\left(\rho-\frac{i \beta_{2}^{\mathrm{Er}}}{2}\right) \frac{\partial^{2} v_{-, 3}}{\partial t^{2}}\right. \\
& +i \gamma\left(v_{-, 3}\left|v_{-, 3}\right|^{2}+A v_{-, 3}\left|u_{-, 3}\right|^{2}\right) \frac{e^{2 g L_{\mathrm{Er}}}-1}{2 g} \\
& \left.+i \gamma B u_{-, 3}^{2} v_{-, 3}^{*} \frac{e^{\left(2 g+4 i n_{\mathrm{Er}}\right) L_{\mathrm{Er}}}-1}{\left(2 g+4 i n_{\mathrm{Er}}\right)}\right] e^{\left(g-i n_{\mathrm{Er}}\right) L_{\mathrm{Er}}}+O\left(\varepsilon^{2}\right),
\end{aligned}
$$

$$
\begin{aligned}
u_{-, 2}= & u_{-, \mathrm{Er}} e^{-i n_{s} L_{2}}+\varepsilon\left[-\frac{i \beta_{2}^{s}}{2} L_{2} \frac{\partial^{2} u_{-, \mathrm{Er}}}{\partial t^{2}}\right. \\
& +i \gamma L_{2}\left(u_{-, \mathrm{Er}}\left|u_{-, \mathrm{Er}}\right|^{2}+A u_{-, \mathrm{Er}}\left|v_{-, \mathrm{Er}}\right|^{2}\right) \\
& \left.+\frac{\gamma B}{4 n_{s}}\left(e^{4 i n_{s} L_{2}}-1\right) v_{-, \mathrm{Er}}^{2} u_{-, \mathrm{Er}}^{*}\right] e^{-i n_{s} L_{2}}+O\left(\varepsilon^{2}\right),
\end{aligned}
$$

$$
\begin{aligned}
v_{-, 2}= & v_{-, \mathrm{Er}} e^{-i n_{s} L_{2}}+\varepsilon\left[-\frac{i \beta_{2}^{s}}{2} L_{2} \frac{\partial^{2} v_{-, \mathrm{Er}}}{\partial t^{2}}\right. \\
& +i \gamma L_{2}\left(v_{-, \mathrm{Er}}\left|v_{-, \mathrm{Er}}\right|^{2}+A v_{-, \mathrm{Er}}\left|u_{-, \mathrm{Er}}\right|^{2}\right) \\
& \left.+\frac{\gamma B}{4 n_{s}}\left(e^{4 i n_{s} L_{2}}-1\right) u_{-, \mathrm{Er}}^{2} v_{-, \mathrm{Er}}^{*}\right] e^{-i n_{s} L_{2}}+O\left(\varepsilon^{2}\right)
\end{aligned}
$$

\section{Propagation along the length $L_{1}$ and $L_{4}$ of standard fiber}

The electric field $\mathbf{E}(0)=\left(u_{0}, v_{0}\right)$ at the entrance of the piece of the standard fiber inserted after the isolator becomes, after propagation over a distance $L_{1}, \mathbf{E}\left(L_{1}\right)=\left(u_{1}, v_{1}\right)$, and is given by

$$
\begin{aligned}
u_{1}= & u_{0} e^{-i n_{s} L_{1}}+\varepsilon\left[-\frac{i \beta_{2}^{s}}{2} L_{1} \frac{\partial^{2} u_{0}}{\partial t^{2}}\right. \\
& +i \gamma L_{1}\left(u_{0}\left|u_{0}\right|^{2}+A u_{0}\left|v_{0}\right|^{2}\right) \\
& \left.+\frac{\gamma B}{4 n_{s}}\left(e^{4 i n_{s} L_{1}}-1\right) v_{0}^{2} u_{0}^{*}\right] e^{-i n_{s} L_{1}}+O\left(\varepsilon^{2}\right), \quad(\mathrm{A} 13) \\
v_{1}= & v_{0} e^{i n_{s} L_{1}}+\varepsilon\left[-\frac{i \beta_{2}^{s}}{2} L_{1} \frac{\partial^{2} v_{0}}{\partial t^{2}}+i \gamma L_{1}\left(v_{0}\left|v_{0}\right|^{2}+A v_{0}\left|u_{0}\right|^{2}\right)\right. \\
& \left.-\frac{\gamma B}{4 n_{s}}\left(e^{-4 i n_{s} L_{1}}-1\right) u_{0}^{2} v_{0}^{*}\right] e^{i n_{s} L_{1}}+O\left(\varepsilon^{2}\right) .
\end{aligned}
$$

After the propagation through length $L_{4}$ of standard fiber, we obtain the electric field $\mathbf{E}_{t}\left(L_{4}\right)=\left(u_{t, 4}, v_{t, 4}\right)$ 
expressed by

$$
\begin{aligned}
u_{t, 4}= & u_{t, 0} e^{-i n_{s} L_{4}}+\varepsilon\left[-\frac{i \beta_{2}^{s}}{2} L_{4} \frac{\partial^{2} u_{t, 0}}{\partial t^{2}}\right. \\
& +i \gamma L_{4}\left(u_{t, 0}\left|u_{t, 0}\right|^{2}+A u_{t, 0}\left|v_{t, 0}\right|^{2}\right) \\
& \left.+\frac{\gamma B}{4 n_{s}}\left(e^{4 i n_{s} L_{4}}-1\right) v_{t, 0}^{2} u_{t, 0}^{*}\right] e^{-i n_{s} L_{4}}+O\left(\varepsilon^{2}\right),
\end{aligned}
$$

$$
\begin{aligned}
v_{t, 4}= & v_{t, 0} e^{i n_{s} L_{4}}+\varepsilon\left[-\frac{i \beta_{2}^{s}}{2} L_{4} \frac{\partial^{2} v_{t, 0}}{\partial t^{2}}\right. \\
& +i \gamma L_{4}\left(v_{t, 0}\left|v_{t, 0}\right|^{2}+A v_{t, 0}\left|u_{t, 0}\right|^{2}\right) \\
& \left.-\frac{\gamma B}{4 n_{s}}\left(e^{-4 i n_{s} L_{4}}-1\right) u_{t, 0}^{2} v_{t, 0}^{*}\right] e^{i n_{s} L_{4}}+O\left(\varepsilon^{2}\right) .
\end{aligned}
$$

[1] F. Ö. Ilday, J. Buckley, L. Kuznetsova, and F. W. Wise, Opt. Express 11, 3550 (2003).

[2] D. Y. Tang and L. M. Zhao, Opt. Lett. 32, 41 (2007).

[3] B. Ortaç, A. Hideur, T. Chartier, M. Brunel, C. Özkul, and F. Sanchez, Opt. Lett. 28, 1305 (2003).

[4] Ph. Grelu, F. Belhache, F. Gutty, and J. M. Soto-Crespo, Opt. Lett. 27, 966 (2002).

[5] F. Amrani, A. Haboucha, M. Salhi, H. Leblond, A. Komarov, Ph. Grelu, and F. Sanchez, Opt. Lett. 34, 2120 (2009).

[6] M. Salhi, H. Leblond, and F. Sanchez, Phys. Rev. A 67, 013802 (2003).

[7] M. Salhi, H. Leblond, and F. Sanchez, Phys. Rev. A 68, 033815 (2003).

[8] H. A. Haus, J. G. Fujimoto, and E. P. Ippen, J. Opt. Soc. Am. B 8, 2068 (1991).

[9] A. Komarov, H. Leblond, and F. Sanchez, Phys. Rev. A 72, 063811 (2005).

[10] Ph. Grelu, F. Belhache, F. Gutty, and J. M. Soto-Crespo, J. Opt. Soc. Am. B 20, 863 (2003).

[11] B. Ortaç, O. Schmidt, T. Schreiber, J. Limpert, A. Tünnermann, and A. Hideur, Opt. Express 15, 10725 (2007).

[12] I. N. Duling III, Opt. Lett. 16, 539 (1991).

[13] M. J. Guy, D. U. Noske, A. Boskovic, and J. R. Taylor, Opt. Lett. 19, 828 (1994).

[14] E. A. Kuzin, B. Ibarra Escamilla, D. E. Garcia-Gomez, and J. W. Hauss, Opt. Lett. 26, 1559 (2001).

[15] B. Ibarra Escamilla, O. Pottiez, E. A. Kuzin, M. Duran-Sanchez, and J. W. Hauss, Laser Phys. 19, 368 (2009).
[16] A. V. Avdokhin, S. V. Popov, and J. R. Taylor, Opt. Express 11, 265 (2003).

[17] J. W. Nicholson and M. Andrejco, Opt. Express 14, 8160 (2006).

[18] N. H. Seong and D. Y. Kim, Opt. Lett. 27, 1321 (2002).

[19] M. J. Guy, D. U. Noske, and J. R. Taylor, Opt. Lett. 18, 1447 (1993).

[20] H. A. Haus, E. P. Ippen, and K. Tamura, IEEE J. Quantum Electron. 30, 200 (1994).

[21] J. Theimer and J. W. Haus, J. Mod. Opt. 44, 919 (1997).

[22] O. Pottiez, B. Ibarra Escamilla, and E. A. Kuzin, Laser Phys. 19, 371 (2009).

[23] M. Salhi, A. Haboucha, H. Leblond, and F. Sanchez, Phys. Rev. A 77, 033828 (2008).

[24] H. Leblond, M. Salhi, A. Hideur, T. Chartier, M. Brunel, and F. Sanchez, Phys. Rev. A 65, 063811 (2002).

[25] G. P. Agrawal, Nonlinear Fiber Optics, 2nd ed. (Academic, New York, 1995).

[26] T. Taniuti and C.-C. Wei, J. Phys. Soc. Jpn. 24, 941 (1968).

[27] H. Leblond, J. Phys. B 41, 043001 (2008).

[28] J. M. Soto-Crespo, N. N. Akhmediev, V. V. Afanasjev, and S. Wabnitz, Phys. Rev. E 55, 4783 (1997).

[29] E. Ding and J. N. Kutz, J. Opt. Soc. Am. B 26, 2290 (2009).

[30] A. J. Stentz and R. W. Boyd, Opt. Lett. 19, 1462 (1994).

[31] B. Ortaç, A. Hideur, M. Brunel, T. Chartier, M. Salhi, H. Leblond, and F. Sanchez, Appl. Phys. B 77, 589 (2003).

[32] N. N. Akhmediev and A. Ankiewicz, Solitons, Nonlinear Pulses and Beams (Chapman and Hall, London, 1997). 